\title{
Low-complexity CFO Compensation for OFDM-based Massive MIMO Systems
}

\author{
Parna Sabeti, Arman Farhang, Nicola Marchetti and Linda Doyle \\ CONNECT / Trinity College Dublin, Ireland \\ Email: \{sabetip, farhanga, nicola.marchetti, ledoyle\}@tcd.ie
}

\begin{abstract}
Massive multiple input multiple output (MIMO) plays a pivotal role in the fifth generation $(5 \mathrm{G})$ wireless networks. However, its performance heavily relies on accurate synchronization. Although timing offset (TO) can be avoided by applying orthogonal frequency division multiplexing (OFDM) with an adequate length of cyclic prefix (CP), carrier frequency offset (CFO) is still a challenging issue. Especially, in the uplink of multi-user massive MIMO systems, CFO compensation can impose a substantial amount of computational complexity to the base station (BS) due to the large number of BS antennas. To resolve this problem, in this paper, we propose a low-complexity CFO compensation technique. Our solution is performed after combining the received signals at the $\mathrm{BS}$ antennas. We calculate the interference matrix and discuss that asymptotically, this matrix can be obtained in terms of the power delay profile (PDP) of the users' channels. Consequently, the computational complexity of the CFO compensation process is independent of the number of BS antennas and thus remains constant as this number increases. Moreover, we show that the calculated interference matrix can be diagonalized due to its circulant property, and as a result, its inverse can be obtained straightforwardly. This leads to a considerable saving in the computational cost of the receiver. Numerical results are presented to verify the performance of our proposed CFO compensation technique and to investigate its computational complexity.
\end{abstract}

\section{INTRODUCTION}

Massive multiple input multiple output (MIMO) is a strong candidate technology for the fifth generation (5G) wireless networks and has drawn noticeable interest from research community, [1], [2]. In a typical massive MIMO system, a large number of antennas, in the order of hundreds, are deployed at the base station (BS), enabling users to simultaneously communicate over the entire available bandwidth. This leads to a significant improvement in the network capacity, [3]. In fact, in massive MIMO channels, noise and multi-user interference are averaged out as a result of the linear combination of the received signals at the BS antennas, [4]. Moreover, emergence of millimeter wave (mmWave) systems, where the large antenna arrays play a crucial role, has highlighted the importance of massive MIMO even further, [5].

Apart from all the advantages, massive MIMO systems may tremendously suffer from synchronization errors, such as timing offset (TO) and carrier frequency offset (CFO), [6], [7]. In orthogonal frequency division multiplexing (OFDM), the effects of TO can be absorbed into a cyclic prefix (CP) providing an adequate guard interval, [8]. However, CFO compensation is still a challenging problem due to the coexistence of multiple-users with different CFOs. Although, there have been plenty of work reported on the frequency synchronization for conventional multi-user OFDM systems, [9]-[11], not all of them are applicable to massive MIMO systems.

In the past decade, some studies have been done to address the synchronization problem for MIMO OFDM systems. Authors in [12], derived an algorithm for joint CFO compensation and multi-user detection for small-scale multi-user MIMO systems. They also considered different complexity levels for the interference cancellation and showed the trade-off between the signal processing effort and the multi-user detection performance through simulations. However, the computational complexity of such an approach is not affordable for largescale MIMO systems. The work in [13] proposes a pilot-based two stage CFO and phase noise (PN) compensation for point to point high frequency MIMO OFDM, where both stages use a conventional least squares (LS) method and need to calculate the inverse of a full rank matrix. In this paper, by referring to other works from literature, [14], [15], inter-carrier interference (ICI) is assumed to be white Gaussian noise to reduce the decoding complexity. Recently, the authors in [16], proposed a massive MIMO frequency synchronization technique that exploits the users' angles of arrival and performs the CFO estimation and compensation for each user individually through a joint spatial-frequency alignment procedure. In order to mitigate the multi-user interference (MUI), they introduced a multi-branch beamforming approach, where they use multiple beamforming vectors for each user. Although this procedure takes place in the time domain, and there should be one receiver per user, the number of required DFT operations per user is equal to the number of branches which is less than the number of BS antennas. Therefore, it has a reduced amount of computational complexity, but it comes at the expense of performance degradation. In fact, when the users are not spatially separated, their angles might be about the same, and the users' signals cannot be accurately distinguished from each other. In [17], an improved user grouping scheme has been designed to deal with this situation, where the CFO estimation and data detection are jointly performed for the users that are close to one another. Despite the imposed computational complexity due to the MUI cancellation, this technique still cannot accurately compensate the CFO effect. This becomes very challenging in dense networks, where many users are in a same area.

In this paper, we propose a new CFO compensation technique for the uplink of OFDM-based multi-user massive MIMO systems, which is performed after combining the received signals at the BS antennas. Here, we employ maximum 
ratio combining (MRC) at the receiver and refer to our proposed technique as Post-MRC. We calculate the interference matrix according to the power delay profile (PDP) of users' channels in an asymptotic regime and discuss that the CFO compensation process can be made independent of the number of BS antennas. In addition, we show that the interference matrix has a circulant property. Thus, it can be diagonalized to simplify the inversion process. As a result, the CFO can be compensated with low computational complexity. Moreover, the compensation process is independent of the number of BS antennas. Thus, as the number of BS antennas increases, the computational complexity related to the CFO compensation remains constant. Finally, we evaluate the performance of our proposed technique through simulations, and demonstrate that its bit error rate (BER) performance matches that of the fully synchronous system when the number of BS antennas is large as compared to the number of users. We also present a thorough complexity analysis for different system parameters, and compare them with that of the trivial time-domain compensation as a benchmark, [18], because of its ideal accuracy and scalability for a large number of antennas and users.

The rest of the paper is organized as follows. Section II presents the system model for the uplink of OFDMbased multi-user massive MIMO system in presence of CFOs. In Section III, we explain our proposed Post-MRC CFO compensation technique. We also calculate the computational complexity of our method and compare it with that of timedomain compensation in Section IV. In Section V, we provide a numerical analysis to investigate the efficacy of the proposed method. Finally, the conclusions are drawn in Section VI.

\section{SYSTEM MODEL}

Consider the uplink of an OFDM system where $P$ single antenna users communicate with a BS having $M \gg P$ antennas. We assume that different users' wireless channels are statistically independent and time invariant during one OFDM symbol. Hence, they can simultaneously share all the available subcarriers as their signals can be distinguished through their respective channel gains. Having $N$ active subcarriers, the $N \times 1$ vector of the $j^{\text {th }}$ OFDM symbol of the $p^{\text {th }}$ user's signal can be obtained as

$$
\mathbf{x}_{p}^{j}=\mathbf{F}_{N}^{\mathrm{H}} \mathbf{d}_{p}^{j},
$$

where the $N \times 1$ vector $\mathbf{d}_{p}^{j}$ contains the $j^{\text {th }}$ data symbol of user $p$ and $\mathbf{F}_{N}$ is the normalized $N$-point DFT matrix with the elements $F_{N}[l, k]=\frac{1}{\sqrt{N}} e^{-j \frac{2 \pi}{N} l k}$ for $l, k=0, \cdots, N-1$. It is worth mentioning that the $i^{\text {th }}$ element of vector a and the $(i, j)^{\text {th }}$ element of matrix $\mathbf{A}$ are denoted as $a[i]$ and $A[i, j]$, respectively. The $\mathrm{CP}$ length is considered to be larger than the channel impulse response (CIR) length, $L$, and the largest user's TO in order to have a perfect time synchronization. In addition, it is assumed that the BS is equipped with coherent oscillators. Thus, the amount of each user's CFO is the same for all the BS antennas. To keep the formulation simple and without loss of generality, we consider only one OFDM symbol. Then, after CP removal, the time domain representation of an OFDM symbol received at the $m^{\text {th }}$ BS antenna can be written as

$$
\mathbf{r}_{m}=\sum_{p=0}^{P-1} \boldsymbol{\Phi}_{p} \mathbf{H}_{m, p} \mathbf{x}_{p}+\mathbf{n}_{m}
$$

where $\mathbf{n}_{m}$ is the complex additive white Gaussian noise (AWGN), i.e., $\mathbf{n}_{m} \sim \mathcal{C} \mathcal{N}\left(0, \sigma_{n}^{2} \mathbf{I}_{\mathrm{N}}\right)$ at the $m^{\text {th }}$ antenna and $\boldsymbol{\Phi}_{p}$ is an $N \times N$ diagonal CFO matrix with the diagonal elements $\Phi_{p}[i, i]=e^{j \frac{2 \pi}{N} i \epsilon_{p}+j \varphi_{p}}$ for $i=0, \cdots, N-1$ in which $\epsilon_{p}$ is the CFO normalized to subcarrier spacing, and $\varphi_{p}$ is the phase shift of $p^{\text {th }}$ user. If the OFDM symbols are transmitted consecutively, $\varphi_{p}$ for $j^{\text {th }}$ symbol is given as $\varphi_{p}^{j}=\varphi_{p}^{j-1}\left(N+N_{C P}-1\right)$ where $N_{C P}$ is the CP length. In addition, $\mathbf{H}_{m, p}=\operatorname{circ}\left(\mathbf{h}_{m, p}\right)$ is a circulant matrix where the first column, $\mathbf{h}_{m, p}$, is the $N \times 1$ CIR vector between the $p^{\text {th }}$ user and the $m^{\text {th }}$ BS antenna that is zero-padded to have the length of $N$ as usually $L<N$. We assume the channel taps to be a set of independent and identically distributed (i.i.d.) random variables that follow a complex normal distribution $\mathcal{C N}(0, \rho(i)), i \in\{0, \cdots, L-1\}$, where $\rho(i), i=0, \cdots, L-1$, is the channel PDP.

After discrete Fourier transform (DFT) operation at the $m^{\text {th }}$ antenna, the received signal in the frequency domain is given by

$$
\overline{\mathbf{r}}_{m}=\sum_{p=0}^{P-1} \mathbf{F}_{N} \boldsymbol{\Phi}_{p} \mathbf{H}_{m, p} \mathbf{x}_{p}+\mathbf{F}_{N} \mathbf{n}_{m} .
$$

Considering $\mathbf{F}_{N}^{\mathrm{H}} \mathbf{F}_{N}=\mathbf{I}_{N}$, equation (3) can be rewritten as

$$
\overline{\mathbf{r}}_{m}=\sum_{p=0}^{P-1} \mathbf{F}_{N} \boldsymbol{\Phi}_{p} \mathbf{F}_{N}^{\mathrm{H}} \mathbf{F}_{N} \mathbf{H}_{m, p} \mathbf{F}_{N}^{\mathrm{H}} \mathbf{d}_{p}+\overline{\mathbf{n}}_{m},
$$

where $\overline{\mathbf{n}}_{m}=\mathbf{F}_{N}^{\mathrm{H}} \mathbf{n}_{m}$ is the frequency domain noise vector. By defining $\mathbf{E}_{p} \triangleq \mathbf{F}_{N} \boldsymbol{\Phi}_{p} \mathbf{F}_{N}^{\mathrm{H}}$ and $\tilde{\mathbf{H}}_{m, p} \triangleq \mathbf{F}_{N} \mathbf{H}_{m, p} \mathbf{F}_{N}^{\mathrm{H}}$, we have

$$
\overline{\mathbf{r}}_{m}=\sum_{p=0}^{P-1} \mathbf{E}_{p} \tilde{\mathbf{H}}_{m, p} \mathbf{d}_{p}+\overline{\mathbf{n}}_{m} .
$$

It is worth noting that due to the circulant property of $\mathbf{H}_{m, p}$, $\tilde{\mathbf{H}}_{m, p}$ is a diagonal matrix, [19], i.e., $\tilde{\mathbf{H}}_{m, p}=\operatorname{diag}\left(\mathbf{F}_{N} \mathbf{h}_{m, p}\right)$.

Deploying the matched filter (MF) detector, the equalizer coefficient at the $k^{\text {th }}$ subcarrier of user $p$ at the $m^{\text {th }}$ BS antennas can be obtained as

$$
z_{m, p}[k]=\frac{\bar{h}_{m, p}^{*}[k]}{\left\|\overline{\mathbf{h}}_{p}[k]\right\|^{2}},
$$

where * stands for conjugate operation, $\overline{\mathbf{h}}_{m, p}$ contains the $N$-point DFT coefficients of $\mathbf{h}_{m, p}$ and $\overline{\mathbf{h}}_{p}[k]=$ $\left[\bar{h}_{0, p}[k], \bar{h}_{1, p}[k], \cdots, \bar{h}_{M-1, p}[k]\right]^{\mathrm{T}}$. As a result, the channel is equalized over each subcarrier through the MRC, and the resulting signal of the $q^{\text {th }}$ user is given by

$$
\overline{\mathbf{y}}_{q}=\sum_{m=0}^{M-1} \mathbf{z}_{m, q} \sum_{p=0}^{P-1} \mathbf{E}_{p} \tilde{\mathbf{H}}_{m, p} \mathbf{d}_{p}+\sum_{m=0}^{M-1} \mathbf{z}_{m, q} \overline{\mathbf{n}}_{m},
$$


where $\mathbf{Z}_{m, p}=\operatorname{diag}\left(\mathbf{z}_{m, p}\right)$. Then, defining an $N \times N$ matrix,

$$
\boldsymbol{\Omega}_{q, p}=\sum_{m=0}^{M-1} \mathbf{Z}_{m, q} \mathbf{E}_{p} \tilde{\mathbf{H}}_{m, p},
$$

the $q^{\text {th }}$ user's signal can be written as

$$
\overline{\mathbf{y}}_{q}=\sum_{p=0}^{P-1} \boldsymbol{\Omega}_{q, p} \mathbf{d}_{p}+\tilde{\mathbf{n}}_{m},
$$

where $\tilde{\mathbf{n}}_{m}=\sum_{m=0}^{M-1} \mathbf{Z}_{m, q} \overline{\mathbf{n}}_{m}$. In the absence of CFO, $\boldsymbol{\Omega}_{q, p}$ becomes a diagonal matrix. However, the presence of CFO makes the matrix $\boldsymbol{\Omega}_{q, p}$ non-diagonal and banded whose offdiagonal elements model the ICI effect. In the next section, we propose a low-complexity technique to deal with this interference.

\section{PRoposed Post-MRC CFO COMPENSATION}

In this section, with the assumption of perfect CFO estimation, we concentrate on the CFO correction process. Note that both $\mathbf{Z}_{m, q}$ and $\tilde{\mathbf{H}}_{m, p}$ are diagonal matrices. Hence, with some manipulations, the interference matrix in equation (8) can be represented as

$$
\boldsymbol{\Omega}_{q, p}=\mathbf{E}_{p} \odot \mathbf{B}_{q, p},
$$

where $\odot$ is the element-wise multiplication and

$$
B_{q, p}[l, k]=\sum_{m=0}^{M-1} Z_{m, q}[l, l] \tilde{H}_{m, p}[k, k],
$$

for $l, k=0, \cdots, N-1$. Moreover, $\mathbf{Z}_{m, q}$ can be written as $\mathbf{Z}_{m, q}=\mathbf{D}_{q}^{-1} \tilde{\mathbf{H}}_{m, q}^{\mathrm{H}}$, where $\mathbf{D}_{q}$ is an $N \times N$ diagonal normalization matrix with elements

$$
\mathbf{D}_{q}=\operatorname{diag}\left\{\left\|\overline{\mathbf{h}}_{q}[0]\right\|^{2},\left\|\overline{\mathbf{h}}_{q}[1]\right\|^{2}, \cdots,\left\|\overline{\mathbf{h}}_{q}[N-1]\right\|^{2}\right\} .
$$

As $M$ increases, according to the law of large numbers, $\mathbf{D}_{q} \rightarrow N \mathbf{I}_{\mathrm{N}}$ and $B_{q, p}[l, k] \rightarrow \mathbb{E}\left\{\tilde{H}_{m, q}^{*}[l, l] \tilde{H}_{m, p}[k, k]\right\}$. Since $\tilde{\mathbf{H}}_{m, p}=\operatorname{diag}\left(\overline{\mathbf{h}}_{m, p}\right), \mathbf{B}_{q, p}$ can be obtained as

$$
B_{q, p}[l, k]=\mathbb{E}\left\{\bar{h}_{m, q}^{*}[l] \bar{h}_{m, p}[k]\right\} .
$$

When $p=q$,

$$
B_{q, q}[l, k]=\mathbb{E}\left\{\sum_{i=0}^{N-1} \sum_{i^{\prime}=0}^{N-1} h_{m, q}^{*}[i] h_{m, q}\left[i^{\prime}\right] e^{-j \frac{2 \pi}{N}\left(k i^{\prime}-l i\right)}\right\},
$$

and since $\mathbf{h}_{m, q}$ is an i.i.d. random vector, $\mathbb{E}\left\{h_{m, q}^{*}[i] h_{m, q}\left[i^{\prime}\right]\right\}=0$ for $i \neq i^{\prime}$, and we have

$$
B_{q, q}[l, k]=\sum_{i=0}^{N-1} \rho_{q}[i] e^{-j \frac{2 \pi}{N}(k-l) i}=\bar{\rho}_{q}[k-l],
$$

where $\overline{\boldsymbol{\rho}}_{q}=\mathbf{F}_{N} \boldsymbol{\rho}_{q}$ contains the $N$-point DFT samples of the channel PDP of the $q^{\text {th }}$ user, and consequently, $\bar{\rho}_{q}[i]=$ $\bar{\rho}_{q}[i-N]$. Hence, we can conclude that $\mathbf{B}_{q, q}$ is a circulant matrix with the first column $\bar{\rho}_{q}[-l]$ for $l=0, \cdots, N-1$. Note that since elements of $\boldsymbol{\rho}_{q}$ are real, we have $\bar{\rho}_{q}[-l]=\bar{\rho}_{q}^{*}[l]$. $\mathbf{E}_{q}=\mathbf{F}_{N} \boldsymbol{\Phi}_{q} \mathbf{F}_{N}^{\mathrm{H}}$ is also a circulant matrix as $\boldsymbol{\Phi}_{q}$ is a diagonal matrix. Accordingly, $\boldsymbol{\Omega}_{q, q}$ derived in equation (10), is a circulant matrix and can be written as $\boldsymbol{\Omega}_{q, q}=\mathbf{F}_{N} \mathbf{Q}_{q, q} \mathbf{F}_{N}^{\mathrm{H}}$, where $\mathbf{Q}_{q, q}$ is a diagonal matrix. Therefore, in order to calculate the inverse of the interference matrix, $\boldsymbol{\Omega}_{q, q}$, we need to calculate the inverse of the diagonal matrix $\mathbf{Q}_{q, q}$, and then, $\boldsymbol{\Omega}_{q, q}^{-1}=\mathbf{F}_{N} \mathbf{Q}_{q, q}^{-1} \mathbf{F}_{N}^{\mathrm{H}}$. Finally, the $q^{\text {th }}$ user's signal can be estimated as

$$
\begin{aligned}
\hat{\mathbf{d}}_{q} & =\boldsymbol{\Omega}_{q, q}^{-1} \overline{\mathbf{y}}_{q} \\
& =\mathbf{d}_{q}+\sum_{\substack{p=0 \\
p \neq q}}^{P-1} \tilde{\boldsymbol{\Omega}}_{q, p} \mathbf{d}_{p}+\boldsymbol{\Omega}_{q, q}^{-1} \tilde{\mathbf{n}}_{m},
\end{aligned}
$$

where $\tilde{\boldsymbol{\Omega}}_{q, p}=\boldsymbol{\Omega}_{q, q}^{-1} \boldsymbol{\Omega}_{q, p}$ is the inter-user interference (IUI) matrix between users $q$ and $p$. Note that since different users' channels are uncorrelated, the elements of $\tilde{\boldsymbol{\Omega}}_{q, p}$ tend to zero as $M$ tends to infinity, and multi-user interference will be averaged out.

Moreover, due to the circulant property of the matrix $\boldsymbol{\Omega}_{q, q}$, calculating its first column, which is the element-wise multiplication of the first columns of the matrices $\mathbf{E}_{p}$ and $\mathbf{B}_{q, p}$, is sufficient to form the rest of this matrix. In addition, since an element-wise multiplication in the frequency domain is equivalent to a circulant convolution in the time domain, the diagonal elements of the matrix $\mathbf{Q}_{q, q}$ can be obtained as

$$
\operatorname{diag}\left(\mathbf{Q}_{q, q}\right)=\operatorname{diag}\left(\boldsymbol{\Phi}_{q}\right) \otimes \operatorname{diag}\left(\hat{\boldsymbol{\rho}}_{q}\right),
$$

where $\hat{\boldsymbol{\rho}}_{q}[i]=\boldsymbol{\rho}_{q}[-i]$, and $\otimes$ denotes circular convolution operation. Therefore, we suggest to take the $q^{\text {th }}$ user's signal, $\overline{\mathbf{y}}_{q}$, to the time domain, multiply it by the diagonal matrix $\mathbf{Q}_{q, q}^{-1}$, and finally, bring it back to the frequency domain. Then, the $q^{\text {th }}$ user's signal can be calculated as

$$
\hat{\mathbf{d}}_{q}=\mathbf{F}_{N} \mathbf{Q}_{q, q}^{-1}\left(\mathbf{F}_{N}^{\mathrm{H}} \overline{\mathbf{y}}_{q}\right),
$$

to save more computational cost. The computational complexity of the proposed CFO compensation technique is calculated in the following section.

\section{Computational Complexity}

In this section, we calculate the computational complexity of the receiver with the proposed Post-MRC CFO compensation according to the number of complex multiplications (CMs). Then, we compare the complexity of our proposed CFO compensation method with the conventional time domain compensation technique, [18], as a benchmark due to its ideal accuracy and scalability for large number of antennas and users.

According to our proposed solution discussed in section III, after $M$ number of $N$-point DFT operators followed by $P$ MRC units, $P$ CFO compensation processes are performed. Hence, the number of CMs at the receiver can be denoted as

$$
C_{1}=M C_{\mathrm{DFT}}+P C_{\mathrm{MRC}}+P C_{\mathrm{com}}^{\mathrm{p}},
$$

where $C_{\mathrm{DFT}}$ is the number of CMs for one DFT operator, which is equal to $\frac{N}{2} \log _{2} N$ if FFT technique is utilized, and 
$C_{\mathrm{MRC}}=2 M N$ is the number of CMs for one MRC detector. In order to calculate the number of $\mathrm{CMs}$ required for the proposed Post-MRC CFO compensation, $C_{\text {com }}^{\mathrm{p}}$, first, we need to obtain the matrix $\boldsymbol{Q}_{q, q}$, which requires $N L$ number of CMs due to the circular convolution process introduced in equation (18).

Afterward, we take $\overline{\mathbf{y}}_{q}$ to the time domain, divide it elementwise by the elements on the main diagonal of $\mathbf{Q}_{q, q}$, and bring it back to the frequency domain. Hence, the number of required CMs for the compensation process is given by

$$
\begin{aligned}
C_{\mathrm{com}}^{\mathrm{p}} & =N L+\left[2 C_{\mathrm{DFT}}+N\right] \\
& =2 \frac{N}{2} \log _{2} N+(L+1) N,
\end{aligned}
$$

and the overall number of $\mathrm{CMs}$ at the receiver is

$$
\begin{aligned}
C_{1} & =M C_{\mathrm{DFT}}+P C_{\mathrm{MRC}}+P C_{\mathrm{com}}^{\mathrm{p}} \\
& =M \frac{N}{2} \log _{2} N+P(2 M N) \\
& +P\left[N \log _{2} N+(L+1) N\right] .
\end{aligned}
$$

Regarding the time domain correction, the received signals on all the BS antennas are multiplied by the compensation vector of the desired user, i.e., $\left[1, e^{-j \frac{2 \pi}{N} \epsilon_{q}-j \varphi_{q}}, \cdots, e^{-j \frac{2 \pi}{N}(N-1) \epsilon_{q}-j \varphi_{q}}\right]$ for $q^{\text {th }}$ user, [18]. Thus, after CP removal, $C_{\mathrm{com}}^{\mathrm{t}}=M N$ number of CMs are required per user to detect an OFDM symbol. Afterward, $P M$ number of $N$-point DFT operations are executed followed by $P$ MRC units. Hence, the overall number of $\mathrm{CMs}$ at the receiver can be calculated as

$$
\begin{aligned}
C_{2} & =P C_{\mathrm{com}}^{\mathrm{t}}+P M C_{\mathrm{DFT}}+P C_{\mathrm{MRC}} \\
& =P M N+P M \frac{N}{2} \log _{2} N+P(2 M N) .
\end{aligned}
$$

Note that unlike conventional time-domain compensation, our proposed compensation technique is independent of the number of BS antennas, $M$. In other words, as $M$ grows, $C_{\mathrm{com}}^{\mathrm{p}}$ remains constant, while $C_{\text {com }}^{\mathrm{t}}$ increases as a linear function of $M$. Consequently, with respect to $M, C_{1}$ is a line with the slope of

$$
N\left(\frac{1}{2} \log _{2} N+2 P\right)
$$

while $C_{2}$ has a slope of

$$
N\left(P \frac{1}{2} \log _{2} N+3 P\right) .
$$

In the next section, a numerical analysis is presented to discuss this comparison more in detail.

\section{NUMERICAL ANALYSiS}

In this section, we confirm our theoretical developments in the previous sections through numerical simulations. We investigate the performance of the proposed Post-MRC compensation technique, presented in section III. In Fig. 1, the bit error rate (BER) curves are depicted for $M=256$ and 512 BS antennas, $P=4$ users and $N=256$ subcarriers. Here, the multipath channel SUI-4, proposed by IEEE802.16 broadband

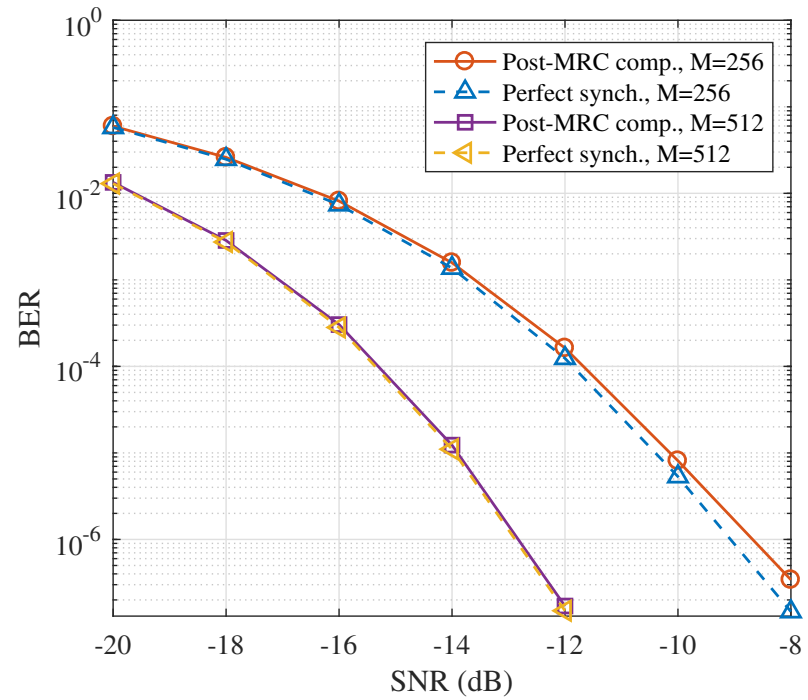

Fig. 1. BER performance of the proposed Post-MRC CFO compensation technique for $P=4$ users and $N=256$ subcarriers.

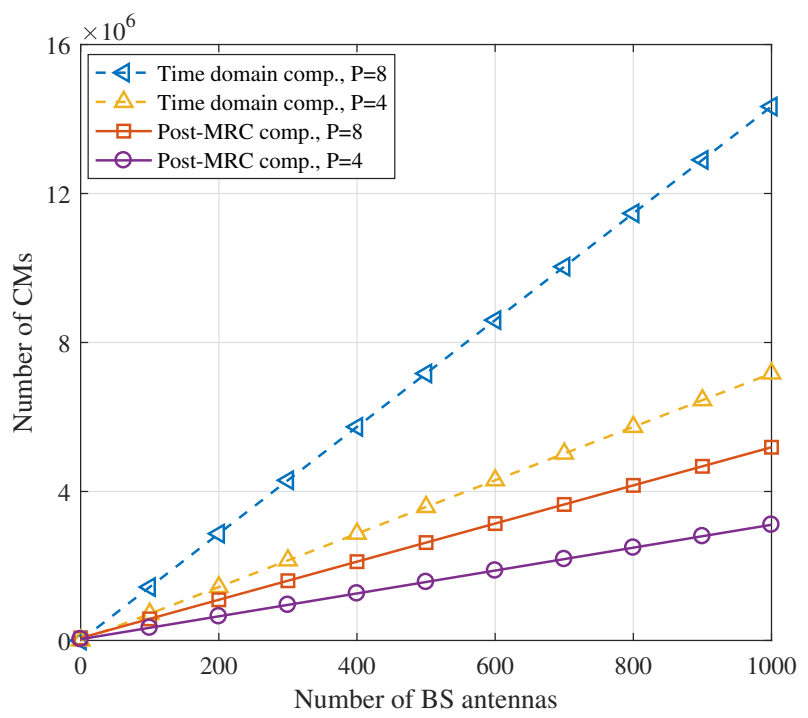

Fig. 2. Number of CMs at the receiver with respect to the number of antennas, $M$, for $N=256$ subcarriers and different number of users, $P$.

wireless access working group [20], is considered, and the CP length is $N / 8$. The normalized CFO is randomly generated from a uniform distribution within the range $[-0.5,0.5)$. We have compared the BER performance of our proposed technique with that of the perfect synchronization as a benchmark. It is shown that the CFO can be sufficiently compensated and the BER curves of Post-MRC method are very close to the benchmarks. As expected, the correction can be more precisely performed as $M$ increases.

In the following, we thoroughly investigate the complexity analysis that was presented in Section IV. The number of CMs at the receiver is calculated for both Post-MRC and timedomain compensation techniques, $C_{1}$ and $C_{2}$, according to 


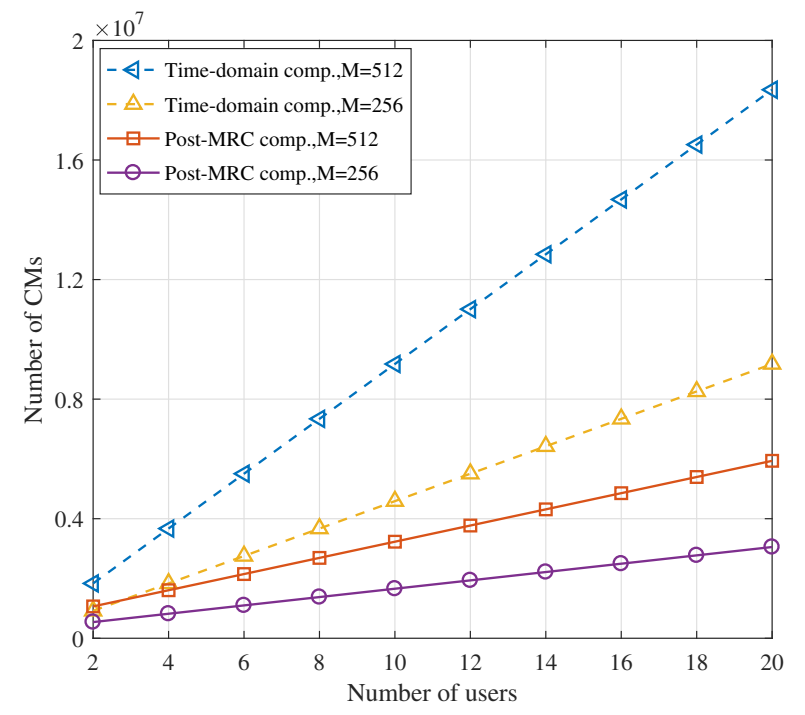

Fig. 3. Number of CMs at the receiver with respect to the number of users, $P$, for $N=256$ subcarriers and different number of BS antennas, $M$.

(21) and (22), respectively. For all the figures, we consider $N=256$ subcarriers and different number of users and antennas. Fig. 2 shows the number of CMs at the receiver with respect to the number of BS antennas. As it is discussed in section IV, unlike time-domain compensation technique, for Post-MRC correction, there is no need for separate receivers per user, which reduces the number of CMs at the receiver. Moreover, Post-MRC correction is independent of the number of BS antennas. This makes $C_{1}$ grow at a slower pace than $C_{2}$, 2.8 and 2.33 times slower for $P=8$ and $P=4$, respectively. Similarly, in Fig. 3, it can be seen that the number of CMs with respect to the number of users for the conventional CFO compensation scheme grow 3.39 and 3.29 times faster than our proposed technique for $M=512$ and $M=256$, respectively.

The number of CMs added to the receiver due to the CFO compensation process is plotted in Fig. 4 to clarify this comparison. As it was pointed out before, the additional CM operations in Post-MRC remain constant as the number of BS antennas increases, while that of the conventional time-domain approach highly depends on the number of BS antennas and tremendously increases with $M$.

To further stress this point, the relative complexity, i.e. the ratio between the number of $\mathrm{CMs}$ at the receiver with $\mathrm{CFO}$ compensation and the receiver with perfect synchronization, is depicted in Fig. 5. As one can see, by applying the timedomain compensation, the number of CMs at the BS is 2.33 and 2.8 times the case of perfect synchronization. While for Post-MRC, this ratio tends to one for very large numbers of BS antennas. This means that the complexity of our Post-MRC compensation technique is negligible compared to the overall complexity of the receiver for large values of $M$.

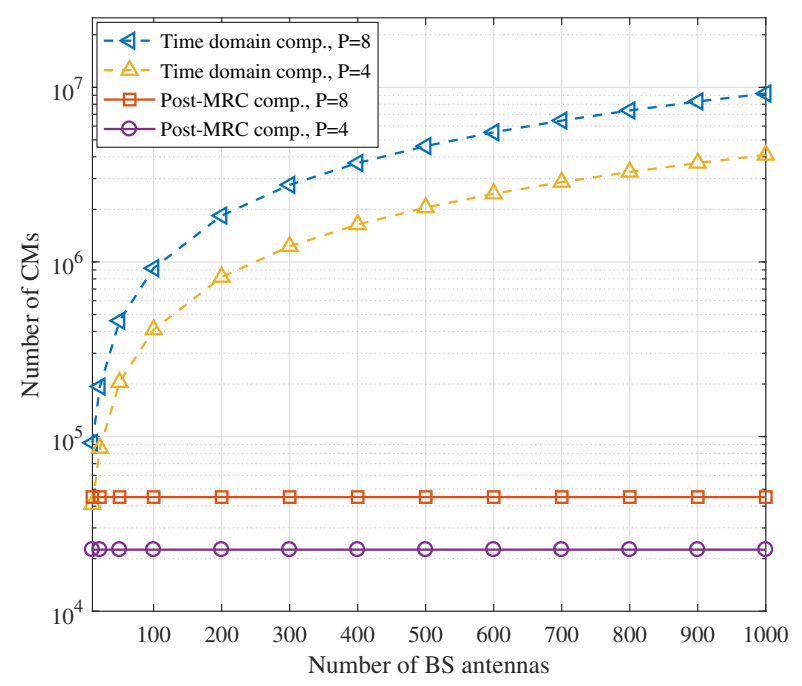

Fig. 4. Number of CMs added to the system due to the CFO compensation process with respect to the number of antennas, $M$, for $N=256$ subcarriers and different number of users, $P$.

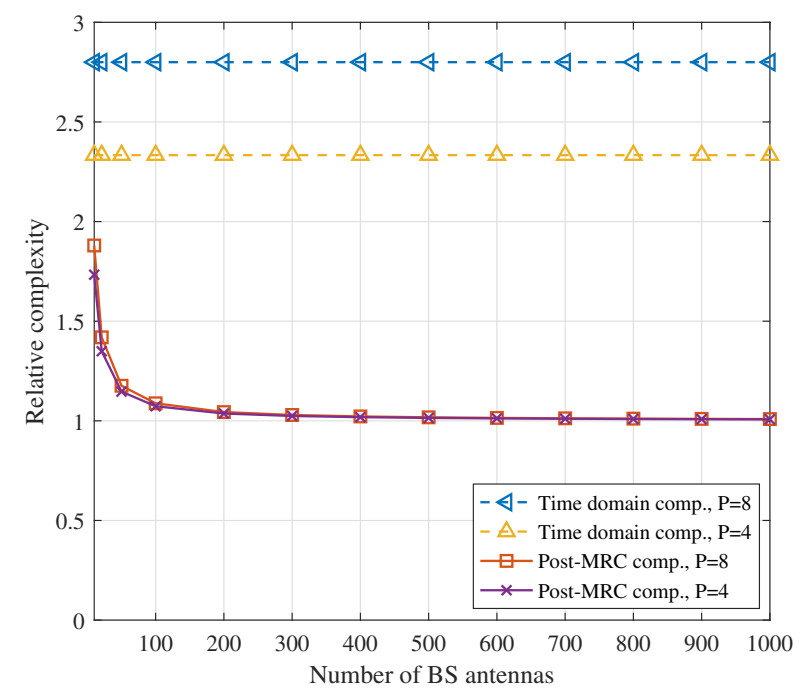

Fig. 5. Relative complexity with respect to the number of BS antennas, $M$, for $N=256$ subcarriers and different number of users, $P$.

\section{CONCLUSION}

In this paper, we proposed a new CFO compensation technique for the uplink of the multi-user OFDM-based massive MIMO systems. We claimed that if the CFO compensation process takes place after combination of the received signals at BS antennas, the interference matrix can be asymptotically obtained in terms of the PDP of the users' channels, and independently of the number of BS antennas. Moreover, we showed that this matrix has a circulant property and its inverse can be calculated straightforwardly. Therefore, our proposed technique is a low-complexity CFO compensation method; in particular, its complexity remains constant when increasing the 
number of BS antennas. This indeed leads to a considerable saving in the computational cost of the receiver of massive MIMO systems. We evaluated the performance of our proposed technique through simulations, and demonstrated when the number of BS antennas is much larger than the number of users, the BER performance of this technique matches that of the fully synchronous system. We also provided a thorough complexity analysis for different system parameters and showed that unlike the time-domain compensation, the complexity of our proposed technique is negligible compared to the overall complexity of the system with large numbers of BS antennas.

\section{ACKNOWLEDGMENT}

This publication has emanated from research conducted with the financial support of Science Foundation Ireland (SFI) and is co-funded under the European Regional Development Fund under Grant Number 13/RC/2077. Moreover, all calculations have performed on the CONNECT cluster maintained by the Trinity Centre for High Performance Computing.

\section{REFERENCES}

[1] J. G. Andrews, S. Buzzi, W. Choi, S. V. Hanly, A. Lozano, A. C. Soong, and J. C. Zhang, "What will 5G be?" IEEE Journal on Selected Areas in Communications, vol. 32, no. 6, pp. 1065-1082, 2014.

[2] E. G. Larsson, O. Edfors, F. Tufvesson, and T. L. Marzetta, "Massive MIMO for next generation wireless systems," IEEE Communications Magazine, vol. 52, no. 2, pp. 186-195, 2014.

[3] F. Rusek, D. Persson, B. K. Lau, E. G. Larsson, T. L. Marzetta, O. Edfors, and F. Tufvesson, "Scaling up MIMO: Opportunities and challenges with very large arrays," IEEE Signal Processing Magazine, vol. 30, no. 1, pp. 40-60, 2013.

[4] T. L. Marzetta, "Noncooperative cellular wireless with unlimited numbers of base station antennas," IEEE Transactions on Wireless Communications, vol. 9, no. 11, pp. 3590-3600, 2010.

[5] J. G. Andrews, T. Bai, M. N. Kulkarni, A. Alkhateeb, A. K. Gupta, and R. W. Heath, "Modeling and analyzing millimeter wave cellular systems," IEEE Transactions on Communications, vol. 65, no. 1, pp. 403-430, 2017.

[6] M. Ganji and H. Jafarkhani, "On the performance of MRC receiver with unknown timing mismatch-a large scale analysis," arXiv preprint arXiv:1703.10422, 2017.

[7] V. Kotzsch and G. Fettweis, "Interference analysis in time and frequency asynchronous network MIMO OFDM systems," in IEEE Wireless Communications and Networking Conference (WCNC), 2010, pp. 1-6.

[8] K. Raghunath and A. Chockalingam, "SIR analysis and interference cancellation in uplink OFDMA with large carrier frequency/timing offsets," IEEE Transactions on Wireless Communications, vol. 8, no. 5 , 2009.

[9] T. M. Schmidl and D. C. Cox, "Robust frequency and timing synchronization for OFDM," IEEE transactions on communications, vol. 45, no. 12 , pp. 1613-1621, 1997.

[10] A. Farhang, N. Marchetti, L. E. Doyle, and B. Farhang-Boroujeny, "Low complexity CFO compensation in uplink OFDMA systems with receiver windowing." IEEE Transactions on Signal Processing, vol. 63, no. 10, pp. 2546-2558, 2015.

[11] H. Minn, V. K. Bhargava, and K. B. Letaief, "A robust timing and frequency synchronization for OFDM systems," IEEE transactions on wireless communications, vol. 2, no. 4, pp. 822-839, 2003.

[12] V. Kotzsch, J. Holfeld, and G. Fettweis, "Joint detection and CFO compensation in asynchronous multi-user MIMO OFDM systems," in IEEE Vehicular Technology Conference. VTC Spring, 2009, pp. 1-5.

[13] H. Huang, W. G. Wang, and J. He, "Phase noise and frequency offset compensation in high frequency MIMO-OFDM system," in IEEE Communications Conference (ICC), 2015, pp. 1280-1285.

[14] K. Y. Kim, Q. Zou, H. J. Choi, and A. H. Sayed, "An efficient carrier phase synchronization technique for high-order M-QAM-OFDM," IEEE Transactions on Signal Processing, vol. 56, no. 8, pp. 3789-3794, 2008.
[15] A. Ishaque and G. Ascheid, "On the efficient mitigation of phase noise in MIMO-OFDM receivers," in IEEE Signals, Systems, and Electronics (ISSSE), 2012 International Symposium on, 2012, pp. 1-6.

[16] W. Zhang, F. Gao, and H.-M. Wang, "Frequency synchronization for massive MIMO multi-user uplink," in IEEE Global Communications Conference (GLOBECOM), 2016, pp. 1-6.

[17] W. Zhang, F. Gao, S. Jin, and H. Lin, "Frequency synchronization for uplink massive MIMO systems," arXiv preprint arXiv:1703.04399, 2017.

[18] S. Mukherjee, S. K. Mohammed, and I. Bhushan, "Impact of CFO estimation on the performance of ZF receiver in massive MU-MIMO systems," IEEE Transactions on Vehicular Technology, vol. 65, no. 11, pp. 9430-9436, 2016.

[19] G. Strang, G. Strang, G. Strang, and G. Strang, Introduction to linear algebra. Wellesley-Cambridge Press Wellesley, MA, 1993, vol. 3.

[20] K. Hari, D. Baum, T. Kaitz, and B. D. Trinkwon, "Project IEEE 802.16 broadband wireless access working group, http://IEEE802. org/16, channel models for fixed wireless applications," 2001. 